\title{
L'accès direct de la personne privée à la juridiction internationale : Une comparaison entre l'arbitrage d'investissement et le contentieux de la Cour européenne des droits de l'homme
}

\author{
Edoardo Stoppioni"
}

\section{Introduction}

Pour le jeune chercheur ayant commencé à étudier le droit à une époque où la Commission du droit international s'interrogeait sur la fragmentation du droit international ${ }^{1}$ et où cette question polarisait fortement les discours doctrinaux, la méthode comparative apparaît comme un outil incontournable pour comprendre l'évolution de l'ordre juridique international. Confronté à un droit international en transformation, passant d'un bric-à-brac à un système organisé, ${ }^{2}$ le chercheur est désormais obligé de penser la complexité de cet ensemble archipélagique. C'est d'autant plus vrai lorsqu'il s'intéresse au droit international économique. Si un premier mouvement a eu tendance à voir dans cette discipline une monade sans portes ni fenêtres sur le droit international général, la volonté de repenser sa nature, grâce à l'instrument comparatif, lui a progressivement succédé. La méthode comparative est donc venue permettre un regard nouveau sur le droit international des échanges et des investissements. ${ }^{3}$

* Research Fellow at the Max Planck Institute Luxembourg for Procedural Law.

1 Rapport du Groupe d'étude de la CDI, Fragmentation du droit international : Difficultés découlant de la diversification et de l'expansion du droit international, 2006, A/CN.4/L.702 qui doit être lu en parallèle avec l'étude analytique établie sous sa forme définitive par M. Koskenniemi, sur laquelle il s'appuie (A/CN.4/L.682 et Corr.1).

2 J. Combacau, Le droit international, bric-à-brac ou système, 31(1) Archives de philosophie du droit (1986), 88.

3 Nous faisons ici référence au débat sur le droit applicable devant le juge de l'OMC, divisant la doctrine entre les partisans de la fermeture du système (J. P. Trachtman, Domain of WTO Dispute Resolution, 40 Harv. Int'l. L.J. (1999), 333.) et son ouverture (J. Pauwelyn, The Role of Public International Law in the WTO : How far can we go?, American Journal of International Law (2001), 535). Le débat s'est également élargi à l'arbitrage d'investissement (A. Pellet, Notes sur la 'fragmentation' du 
Toutefois, ce type d'approche a davantage rayonné pour la compréhension des aspects matériels que pour celle des versants procéduraux du droit. Un vaste corps de littérature est consacré à la question des interactions normatives entre le droit international économique et les autres îles de l'archipel, phénomène identifié par la doctrine anglophone comme un débat sur les 'linkages, ${ }^{4}$ alors que c'est avec une moindre intensité que l'outil comparatif a été employé aux fins d'une mise en abîme de la dimension contentieuse de la matière. Dans cette seconde optique, nous allons nous intéresser à la première étape de la procédure contentieuse, la saisine. ${ }^{5}$

Pour ce faire, l'attention sera principalement portée sur le modèle le plus iconoclaste de saisine en droit international public, celui qui permet l'accès direct de la personne privée à la juridiction internationale, qui n'est plus cantonnée au tout-étatique du contentieux classique. En laissant de côté le contentieux de la fonction publique, opposant un individu à une organisation internationale ${ }^{6}$ ou le contentieux des juridictions supranationales répondant à une logique d'intégration, ${ }^{7}$ il s'agira ici de comparer l'arbitrage d'investissement avec le contentieux international de protection des droits de l'homme, ${ }^{8}$ deux domaines où la personne privée est habilité à sai-

droit international : Droit des investissements internationaux et droits de l'homme, in Unité et diversité du droit international (2014), 757).

4 Il suffit de penser au mouvement 'trade and .., ayant été développé par mimétisme dans la doctrine 'investment and ..., qui ont croisé les problématiques liées au droit international économique avec les droits de l'homme et le droit de l'environnement notamment. Voir notamment T Cottier et al. (dir.), Human Rights and International Trade (2005), et P-M Dupuy et al. (dir.), Human Rights in International Investment Law and Arbitration (2009).

5 Pour une analyse théorique, E. Jouannet, La saisine en droit international ou la simplicité dans la diversité, in H. Ruiz Fabri, J.-M. Sorel, La saisine des juridictions internationales (2006), 307.

6 Pour une analyse fouillée de la jurisprudence en la matière, voir A.-M. ThévenotWerner, Le droit des agents internationaux à un recours effectif. Vers un droit commun de la procédure administrative internationale, thèse, Paris 1 (2014).

7 Dans ce cadre, la logique contentieuse est fort différente et s'écarte du modèle de justice consensuelle qui servira de fond à la comparaison. Voir néanmoins pour un excellent tableau P. Cassia, Laccès des personnes physiques ou morales au juge de la légalité des actes communautaires (2002), XII-1042.

8 Cette analyse n'a pas pris la Cour interaméricaine des droits de l'homme comme terme de comparaison constant car seuls les États membres ou la Commission peuvent saisir la Cour directement (article 61 de la Convention interaméricaine). Ainsi, aux fins d'une analyse de la nature du mécanisme d'accès direct de l'individu à la juridiction, sa jurisprudence constitue un élément moins pertinent. Néanmoins, cette dernière a retenu une lecture extrêmement progressiste du droit de 
sir directement la juridiction internationale du fait d'une violation du droit international dans son chef par un État.

Théoriser la comparaison. Avant de procéder à cette analyse, encore faut-il interroger la pertinence de la méthode qu'il est envisagé d'utiliser. Il est bien connu qu'un important débat a polarisé les points de vue concernant le but ultime du droit comparé. En schématisant, on peut identifier deux courants : pour certains, le droit comparé ne servirait en réalité qu'à décrire la structure des systèmes observés ${ }^{9}$ pour d'autres, il constitue davantage une manière d'" identifier le droit " ${ }^{10}$ une "voie de connaissance critique ${ }^{11}$ destinée à saisir la " complexité réelle du juridique ${ }^{12}$

Bien plus qu'à décrire la structure des deux contentieux observés, l'emploi de la méthode comparative sert ici à saisir les différences et les ressemblances qui animent la dynamique du droit international économique et du droit international des droits de l'homme, la manière dont leurs postulats idéologiques rejaillissent sur les procédures qui les réalisent. Les deux branches semblent en effet trouver leur origine commune dans la pensée libérale. ${ }^{13}$ Il n'est pas anodin que les écoles critiques du droit international,

participation de l'individu à l'instance, comme le souligne A. A. Cançado Trindade, The Access of Individuals to international justice (2011), 37. De même, n'a pas été retenue comme tertium comparationis systématique la jurisprudence des Comités des Nations Unis, dont le caractère juridictionnel reste débattu mais surtout dès lors que l'accès de l'individu fonctionne à géométrie variable, selon la ratification par les États du protocole additionnel le prévoyant.

9 O. Pfersmann, Le droit comparé comme interprétation et comme théorie du droit, 53(2) Revue internationale de droit comparé (2001), 275.

10 E. Picard, L'état du droit comparé en France, en 1999, 51(4) Revue internationale de droit comparé (1999), 885.

11 H. Muir Watt, La fonction subversive du droit comparé, 52(3) Revue internationale de droit comparé (2000), 503.

12 M.C. Pontherau, Le droit comparé en question(s). Entre pragmatisme et outil épistémologique, 57(1) Revue internationale de droit comparé (2005), 7.

13 E. Tourme-Jouannet, Le libéralisme économique du droit international contemporain : entre objectifs keynésiens et triomphe du libre-échange, in Le droit international libéral-providence : une histoire du droit international (2011), 285-294 : «On retrouve donc en matière économique les implications du tournant contemporain du droit international vers le libéralisme politique de la démocratie, de l'État de droit et des droits de l'homme que nous avons évoqués plus haut. Il n'y a pas de surprise en cela sachant que le libéralisme politique et le libéralisme économique sont intimement liés selon nous, comme on l'a dit à plusieurs reprises au cours de cet ouvrage, dès lors que l'on retrouve dans certains droits et libertés du libéralisme politique les principes qui soutiennent le libéralisme économique qui n'est pas le capitalisme - comme la liberté de commerce, la propriété privée ou la liberté d'entreprendre ». 
en raison de leur postulat de départ antilibéral, émettent des réserves aussi bien sur le discours du droit international économique que sur celui des droits de l'homme. ${ }^{14}$ À ce propos, Anne Orford considère que "focusing on this question of the forms of law embodied in the two fields of trade and human rights is helpful, perhaps even necessary, in developing an understanding of the relationship between liberal democratic politics and global capitalist economics $»^{15}$, considérations pouvant également être étendues au domaine de l'investissement.

Si l'on fait appel à l'histoire des idées, l'origine commune des droits humains et du droit international économique est plus ancienne qu'on le pense généralement. Comme l'a montré Max Weber, dès le $\mathrm{X}^{\mathrm{ème}}$ siècle, les Républiques maritimes italiennes ont commencé à attribuer aux marchands un droit de citoyenneté du fait, non plus de leur participation à la guerre, mais de leur contribution à l'activité économique de l'État. ${ }^{16} \mathrm{La}$ seconde scolastique du XVİ̀me siècle a continué à creuser ce sillon. Dans son De Indis, Francisco De Vitoria justifie l'existence d'un droit au commerce inoffensif de l'étranger par le fait que le ius gentium interdit de maltraiter l'individu qui ne cause aucun mal à autrui, interdiction dont la ratio legis est située dans le respect de tout être humain imposé par le droit divin. ${ }^{17}$ On voit bien que, dans un premier temps, la reconnaissance de certains droits de l'individu est entremêlée à l'activité économique de l'étranger et, inversement, que la liberté du commerce dépend des premiers. Dans ce même registre, les premiers penseurs de la libéralisation des échanges ancrent son exigence dans l'idée d'une paix par le commerce favorisant l'épanouissement humain. ${ }^{18}$

14 R. Bachand, Les théories critiques du droit international aux États-Unis et dans le monde anglophone (2015), 12; R. M. Unger, The critical legal studies movement, 96 Harvard Law Review (1983), 561-675.

15 A. Orford, Beyond Harmonization : Trade, Human Rights and the Economy of Sacrifice, 18(2) Leiden Journal of International Law (2005), 180.

16 M. Weber, La ville (2014).

17 F. De Vitoria, De Indis et De Iure Belli Relectiones, texte de 1696 (E. Nys dir., 1917). Relectio I, section III, 386 : « Sic enim apud omnes nationes habetur in humanum, sine aliqua speciali causa hospites et peregrinos male accipere : contrario autem humanum et officiosum, se habere bene erga hospites : quod non esset, si peregrini male facerent ».

18 Montesquieu, De l'esprit des lois (1748), Livre XX, Chapitres I et II : "Le commerce guérit des préjugés destructeurs; et c'est presque une règle générale que partout où il $\mathrm{y}$ a des mœurs douces il $\mathrm{y}$ a du commerce, et que partout où il $\mathrm{y}$ a du commerce il y a des mœurs douces. [...] L'effet naturel du commerce est de porter à la paix. Deux nations qui négocient ensemble se rendent réciproquement dé- 
Néanmoins, la réalisation juridique de ces prémisses idéologiques prend des chemins plus tortueux. Il ne fait pas de doute que la protection internationale de l'opérateur économique précède celle de l'être humain, dès lors que le standard minimum de traitement s'est cristallisé bien avant l'internationalisation de la protection des droits humains. Ces obligations coutumières étaient moins ancrées dans l'idée de protéger l'homme que dans la vision westphalienne d'un lien inextricable entre l'étranger et son État d'appartenance. ${ }^{19} \mathrm{Ce}$ contraste se reflète dans l'architecture du droit international classique. D’un point de vue matériel, les traités de paix, commerce et navigation sont ancrés dans la réciprocité, ${ }^{20}$ dans le $d o$ ut des qui rappelle la logique du compromis économique. ${ }^{21}$ D'un point de vue procédural, l'institution de la protection diplomatique permet de mettre à nu le fondement viscéralement interétatique du recours juridictionnel dont bénéficiait l'étranger.

On assiste toutefois à une rupture progressive avec cette logique. La réciprocité comme base classique du droit des traités est remise en cause, d'abord avec la montée d'instruments de protection des droits de l'homme et de droit humanitaire, qui sont davantage ancrés dans l'exigence de symétrie morale kantienne que dans une logique contractualiste. ${ }^{22}$ Parallèlement, sur le plan contentieux, toute une kyrielle d'instruments vient pro-

pendantes : si l'une a intérêt d'acheter, l'autre a intérêt de vendre; et toutes les unions sont fondées sur des besoins mutuels".

19 P.-M. Dupuy, J. Viñuales, Human Rights and Investment Disciplines : Integration in Progress, in M. Bungenberg et al., International Investment Law (2015), 1739, 1743.

20 E. Decaux, La réciprocité en droit international (1980); M. Virally, Le Principe de Réciprocité dans le Droit International Contemporain, t. 122 RCADI (1967).

21 Commission de conciliation franco-mexicaine, Georges Pinson c. États-Unis du Mexique, 19 octobre 1928, RSA, vol. V, 341.

22 TPIY Procureur c. Zoran Kupreskic, Mirjan Kupreskic, Vlatko Kupreskic, Drago Josipovic, Dragan Papic, Vladimir Santic, 14 janvier 2000, $\$ 518$ : " Le caractère absolu de la plupart des obligations prévues par les règles du droit international humanitaire vient de la tendance progressive à l' 'humanisation' des obligations de droit international, qui s'illustre par le recul généralisé du rôle de la réciprocité dans l'application du droit humanitaire au cours de ce dernier siècle. Après la Première Guerre mondiale, l'application du droit de la guerre s'est écartée du concept de réciprocité entre les belligérants, ce qui fait qu'en général les règles ont de plus en plus été appliquées par chacun d'entre eux indépendamment de l'éventualité que l'ennemi ne les respecte pas. Ce changement de perspective vient de ce que les États ont pris conscience que les normes du droit international humanitaire avaient avant tout pour vocation, non de protéger leurs intérêts, mais ceux des personnes en leur qualité d'êtres humains. À la différence d'autres normes internationales, comme celles portant sur les traités commerciaux qui peuvent légitime- 
gressivement mettre en place des voies de recours directes de la personne privée à la juridiction internationale. Dans quelle mesure, alors, cette évolution du mécanisme de saisine témoigne-t-elle réellement d'une évolution paradigmatique?

Théoriser l'action. D'un point de vue plus technique, la question de l'accès à la juridiction a fait l'objet d'une longue tentative de théorisation en droit processuel. Le point de départ de cette réflexion se trouve, sans surprise, dans le droit romain et sa conception de l'actio, définie par Celse comme "nihil aliud quam ius persequendi in iudicio, quod sibi debetur ${ }^{23}$ Telle est l'origine de l'idée selon laquelle l'action a précédé intellectuellement la notion de droit substantiel, le droit romain ayant reposé sur une batterie d'actions (in rem, in personam) plutôt que sur des droits subjectifs.

Ce n'est qu'une fois que la théorie des droits subjectifs a investi celle du contentieux, que les théoriciens du procès repensent la nature de l'actio en droit romain, afin de la transposer au droit commun. Selon la position traditionnelle (dite aussi subjectiviste ou moniste), prônée par Savigny, l'action ne serait pas autre chose que le droit substantiel mis en mouvement. Il y aurait donc identité entre le droit procédural d'agir en justice et le droit substantiel revendiqué sur le fond. ${ }^{24}$ À cela s'oppose la théorie de l'autonomie (dite également objectiviste ou dualiste), inaugurée par Windscheid. ${ }^{25}$ Selon celle-ci, l'actio est désormais séparée du droit substantiel invoqué au fond et est vue comme un droit subjectif autonome (le Klagerecht). La justification de cette scission est bien connue : il existe en effet des droits sans action (comme dans le cas des obligations naturelles) tout comme des actions sans droit (comme dans le cas du contentieux objectif ou de l'action pénale). Cette dissociation doit s'analyser de manière différente selon le contexte : un grand nombre d'ordres juridiques nationaux voit s'affirmer un droit fondamental d'accès au juge; alors que, dans le contentieux international, le principe de justice consensuelle, constamment réitéré par les

ment se fonder sur la protection des intérêts réciproques des États, le respect des règles humanitaires ne peut dépendre d'un respect réciproque ou équivalent de ces obligations par d'autres États. Cette tendance inscrit dans les normes juridiques le concept 'd'impératif catégorique', formulé par Kant dans le domaine de la morale : il convient de s'acquitter de ses obligations, que les autres le fassent ou non ».

23 Instututiones Iustinianeae, 4.6 De actionibus : " rien d'autre que le droit de poursuivre en jugement ce qui nous est dû » (notre traduction).

24 F. C. von Savigny, System des heutigen römischen Rechts (1841), vol. V, para 204.

25 B. Windscheid, Die Actio des römischen Civilrechts, vom Standpunkte des heutigen Rechts (1856), para 23. 
deux juridictions permanentes, ${ }^{26}$ a sécrété l'idée selon laquelle ce n'est pas parce qu'un État s'est engagé à prévoir un droit substantiel qu'il a également accepté le règlement juridictionnel des différends qui en découlent. ${ }^{27}$

On perçoit dans les divisions doctrinales de théorie du procès une gradation d'analyse possible en ce qui concerne la texture juridique de l'action. Celle-ci peut être conçue comme un droit subjectif concret, consistant en un droit à obtenir une décision; ${ }^{28}$ mais elle peut également être vue comme une simple faculté procédurale, un droit abstrait et complètement déconnecté de la substance du droit revendiqué au fond, un simple droit de provoquer l'exercice de la juridiction. ${ }^{29}$ On peut donc se demander si ces différentes nuances ne correspondent pas à des compréhensions différentes que la juridiction se fait du mécanisme de recours, permettant aussi d'analyser les deux modalités d'accès direct à la juridiction internationale.

Dans le discours juridictionnel international sur la saisine, deux pôles peuvent être identifiés. D'une part, une perspective volontariste conduit à situer la source de la faculté de saisine dans la volonté de l'État d'adhérer à l'instrument de protection internationale ménageant une voie contentieuse. D'autre part, une perspective davantage objectiviste rattache l'existence du droit d'action à l'exigence d'effectivité d'une position juridique méritant protection. Des éléments des deux pôles discursifs sont présents dans les deux contentieux étudiés. Le discours volontariste se retrouve, sans surprise, dans l'arbitrage international, mécanisme laissé à la volonté des parties. De manière similaire, les États membres du Conseil de l'Europe n'ont eu l'obligation d'aménager un accès direct à la Cour qu’à partir de l'entrée en vigueur du protocole $\mathrm{n}^{\circ} 11$. En revanche, le discours objectiviste renvoie davantage à une philosophie voyant dans les droits humains des objets de valeur universelle ${ }^{30}$ et qui prône, avant tout, une exigence de pro-

26 Statut de la Carélie orientale, avis du 23 juillet 1923, CPJI, série B, p. 27; CIJ, Affaire de l'or monétaire pris à Rome en 1943 (question préliminaire), arrêt du 15 juin 1954, CIJ Rec. 1954, 32.

27 Activités armées sur le territoire du Congo (nouvelle requête : 2002) (République démocratique du Congo c. Rwanda), compétence et recevabilité, arrêt du 3 février 2006, CIJ Rec. 2006, \$ 64.

28 A. Wach, Handbuch des deutschen Civilprozessrechts (1885), 21.

29 A. Plósz, Beiträge zur Theorie des Klagerechts (1882), 121. Une position similaire sera reprise par différents auteurs (notamment Rocco, Carnelutti, Degenkolb ou encore Liebman).

30 J. Raz, Human Rights in the Emerging World Order, 1 Transnational Legal Theory (2010), 39-41. 
tection de ces droits qui ne soit pas illusoire, mais concrète et effective. ${ }^{31} \mathrm{Il}$ renvoie également à l'idée d'un investisseur considéré, dans le Zeitgeist d'une société marchande, comme un 'sujet sacré' et méritant d'être protégé en raison de sa 'fonction sociale. ${ }^{32}$ On glisse ainsi d'un premier discours cantonnant la saisine au corollaire de la volonté de soumission de l'État à la juridiction, à un langage d'attribution inhérente à cet homo sacer, à un droit qui lui revient en raison de son identité même.

Geneviève Bastid Burdeau avait considéré en 1995 que les systèmes de l'arbitrage d'investissement et celui du contentieux qui se développe devant la Cour européenne des droits de l'homme méritent d'être comparés, dès lors qu'ils prévoient tous deux un droit de recours direct pour des particuliers non identifiés à l'avance, relevant de la "même philosophie " bien que présentant des "différences évidentes et importantes ". ${ }^{33}$ L'idée que nous nous proposons ici de défendre est celle de la différence de nature entre l'accès direct de l'investisseur au tribunal arbitral et celui du requérant individuel à la juridiction de protection des droits de l'homme. En dépit d'une pluralité d'éléments discursifs convergents dans la jurisprudence, les deux juridictions internationales ont globalement compris l'accès direct de la personne privée de manière distincte. La Cour européenne des droits de l'homme a progressivement façonné un véritable droit d'action individuel,

31 Airey c. Irlande, CEDH Numéro 6289/73, arrêt du 9 octobre 1979, $\mathbb{} 24$ : « la Convention a pour but de protéger des droits non pas théoriques ou illusoires, mais concrets et effectifs ».

32 M. Chemillier-Gendreau, Droit international et démocratie mondiale (2002), 68-69, selon qui les accords de protection des investisseurs étrangers sont " au cœur des dangers dont le capitalisme dans sa phase actuelle menace nos sociétés ", parvenant à "interdire aux États de légiférer à l'égard des investissements étrangers, même pour protéger leur société nationale contre les avancées des multinationales et contre les menaces qu'elles font peser sur la santé, l'emploi, l'environnement ». Il s'agit du «symbole de la nécessité pour le capitalisme de détruire la loi et d'imposer dans la symbolique usurpée d'une loi internationale, le mécanisme de destruction de la potentialité de la loi interne ».

33 G. Bastid-Burdeau, Nouvelles perspectives pour l'arbitrage dans le contentieux économique intéressant les États, 1 Revue de l'arbitrage (1995), 3, 15 : «En réalité le rapprochement paraît davantage s'imposer avec le mécanisme de recours individuel prévu par la Convention européenne des Droits de l'Homme, en dépit des différences évidentes et importantes qui existent entre les deux systèmes. Mais la " philosophie » des deux mécanismes paraît la même : il s'agit dans l'un et l'autre cas d'ouvrir à des particuliers non identifiés à l'avance un droit de recours direct contre un État en vue de sanctionner le respect de l'engagement pris par ce dernier dans un traité international d'accorder un certain traitement à des personnes privées ». 
participant à la logique même de la Convention et essentiel pour son fonctionnement, un droit procédural fondamental de la personne soumise à la juridiction d'un État membre. En revanche, il n'existe pas de droit inhérent de l'investisseur à la saisine du tribunal arbitral. Un tel droit ne saurait être conçu comme un droit humain, mais bien plus comme une faculté procédurale (de provoquer l'exercice de la juridiction) reconnue à un opérateur économique situé et dépendant strictement des limites du consentement de l'État. On ne saurait donc parler dans ce cadre d'un véritable droit d'action individuelle. ${ }^{34}$

La différence de nature des recours s'impose puisque, comme le constate la théorie du procès, à des exigences de protection différentes correspondent des formes de protection différentes. ${ }^{35}$ Il sera donc démontré, dans un premier temps, que ces différences tiennent à l'évolution conceptuelle des deux contentieux, à une évolution divergente de leurs postulats de base (I - La perspective diachronique), avant de montrer comment ce décalage idéologique se reflète dans la pratique des juridictions internationales, qui a façonné deux typologies d'accès direct extrêmement divergentes (II - La perspective synchronique).

\section{La perspective diachronique}

En opérant une déconstruction historique de la réflexion sur la nature des mécanismes d'accès direct de la personne privée à la juridiction internationale, on constate que son analyse a longtemps été placée en second plan dans le discours doctrinal (A). Néanmoins, en comparant les travaux préparatoires de la Convention de Washington et de la Convention de sauvegarde des droits de l'homme et des libertés fondamentales, une divergence d'optique apparaît (B).

34 Contra D. Burriez, Le droit d'action individuelle sur le fondement des traités de promotion et de protection des investissements, thèse, Paris 2 (2014), 479 : "Ces termes semblent décrire ce droit d'obtenir du tribunal une décision sur le fond, que le droit judiciaire appelle droit d'agir en justice. Il s'est agi dans cette étude d'apprécier cette évolution sur le terrain du droit afin de discuter l'utilité du concept pour le droit du contentieux international. L'étude a permis de la confirmer à plusieurs égards ".

35 H. Motulsky, Le droit subjectif et l'action en justice, Archives de Philosophie du Droit (1964), 215; A. Proto Pisani, Tutela giurisdizionale differenziata e nuovo processo del lavoro, V Foro italiano (1973), 205. 


\section{A. Une diversité éclipsée}

Si l'on essaie de retracer une histoire intellectuelle de l'accès direct de la personne privée à la juridiction internationale, on remarque d'emblée que les analyses de la nature procédurale de ce mécanisme n'abondent guère. Cela peut susciter l'étonnement, compte tenu du fait que, déjà en 1929, l'Institut de droit international s'était intéressé à ce sujet. ${ }^{36}$ Cette quiétude doctrinale tient au fait que la question de l'accès direct a été reléguée au second plan, ayant toujours été asservie à la réflexion sur la subjectivité de l'individu en droit international (1). En revanche, les deux mécanismes d'accès, celui de l'investisseur et celui du contentieux des droits de l'homme, ont une généalogie intellectuelle différente qu'il convient de mettre en lumière (2).

\section{La question de l'accès direct éclipsée par le débat sur la subjectivité}

Le discours doctrinal relatif au statut procédural de la personne en droit international est marqué par une certaine logique circulaire : l'individu n'a pas à avoir accès direct à la juridiction internationale puisqu'il n'est pas un sujet de droit international, ce qui est le revers du fait que l'individu n'est pas devenu sujet de droit international puisque, inter alia, il n'a pas encore de capacité procédurale dans l'ordre juridique international. ${ }^{37}$

Lécole positiviste, qui a structuré la réflexion sur le droit international à compter des XVIII et XIX ${ }^{\text {èmes }}$ siècles, avait fait de l'individu un simple objet du droit des relations interétatiques. ${ }^{38}$ Cette posture mène à deux interprétations particulières. D'un côté, les traités sont conçus purement et simplement comme des accords entre États et, même lorsqu'ils semblent accorder des droits directement aux individus, ils ne font en réalité qu'obliger les

36 Résolution concernant le problème de l'accès des particuliers à des juridictions internationales, IDI Session de New York, 16 octobre 1929, Rapporteur Stelio Séfériadès : «L'Institut de Droit international est d'avis qu'il y a des cas dans lesquels il peut être désirable que le droit soit reconnu aux particuliers de saisir directement, sous des conditions à déterminer, une instance de justice internationale de leurs différends avec des Etats ».

37 Voir sur la complexité de penser la catégorie du sujet la brillante analyse d'H. Ruiz Fabri, Les catégories de sujet du droit international, in SFDI Colloque du Mans, Le sujet en droit international (2005), 55-71.

38 B. Taxil, Recherches sur la personnalité juridique internationale : l'individu, entre ordre interne et ordre international, thèse, Paris 1 (2005), 65. 
États les leur conférer par le truchement de l'ordre interne. D’un autre côté, la faculté de demander l'exécution de l'obligation conventionnelle n'existe que dans les relations entre États contractants; quand bien même le traité organiserait un recours devant un organe juridictionnel international, celui-ci constitue un droit de nature purement instrumentale. ${ }^{39}$

Les exceptions à l'omniprésence de l'État-requérant dans le contentieux international de l'avant-Seconde Guerre mondiale sont négligées par la doctrine qui ne cesse d'en souligner le caractère ponctuel, sui generis, au surplus régionalement circonscrit. ${ }^{40}$ On préfere donc utiliser les outils procéduraux permettant à l'individu de réclamer dans l'ordre juridique international en tant que moyen de preuve de l'absence de personnalité juridique de celui-ci. Les mécanismes procéduraux permettant l'accès direct de l'avant-Première Guerre mondiale, qu'il s'agisse de la Cour de justice centre-américaine ${ }^{41}$ ou de la proposition d'une Cour internationale des prises $^{42}$ ont reçu peu d'attention. De même, les mécanismes tribunaux arbitraux mixtes de l'après Première Guerre mondiale ${ }^{43}$ ou l'expérience de la Haute Silésie ${ }^{44}$ sont quasiment absents des manuels de l'époque. ${ }^{45}$ On peut néanmoins rappeler la position d'Anzilotti, niant aux tribunaux arbitraux mixtes la nature même de juridictions internationales et préférant y voir des tribunaux étatiques communs, un organe commun vivant en même temps dans les deux ordres juridiques nationaux, ${ }^{46}$ opinion suivie notamment par Sperduti, selon lequel il s'agit simplement d' " organes interna-

39 R. Quadri, La sudditanza nel diritto internazionale (1936), 58.

40 F. A. von der Heydte, L'individu et les tribunaux internationaux, t. 107 RCADI (1962), 318 ; F. Capotorti, Cours général de droit international public, t. 248 RCADI (1994), 85-90.

41 Voir néanmoins M. O. Hudson, The Central American Court of Justice, 26(4) The American Journal of International Law (1932), 759-786.

42 Principale exception est la thèse de L. Katz, Der internationale Prisenhof (1910).

43 Remarquable à ce propos R. Blühdorn, Le fonctionnement et la jurisprudence des Tribunaux Arbitraux Mixtes créés par les Traités de Paix, 41 RCADI (1932), 137-244.

44 G. Kaeckenbeeck, The international experiment of Upper Silesia : a study in the working of the Upper Silesian settlement, 1922-1937 (1942).

45 M. Erpelding, Upper Silesian Mixed Commission, MPILux Working Paper 5 (2017).

46 D. Anzilotti, Corso di diritto internazionale (1926), 163 : « i tribunali arbitrali misti istituiti in conformità all'art. 304 del trattato di Versailles (e disposizioni corrispondenti degli altri trattati di pace) in quanto decidono controversie fra privati o fra questi e lo stato, sono tribunali costituiti, nell'uno a nell'altro dei due ordinamenti giuridici : ma identica essendone in ogni caso la composizione, identiche le norme secondo cui procedono, assunte come proprie da ognuno dei due Stati me- 
tionaux de justice interne ", constitués sur la base de normes internationales, mais fonctionnant à partir de normes de droit interne. ${ }^{47}$

Ce type d'analyse positiviste imprègne les travaux ayant abouti à la création des premières juridictions internationales. Au sein du 'Comité des dix' auteurs du Statut de la Cour permanente de Justice internationale (CPJI), Ricci Busatti affirmait notamment qu' « il est impossible de mettre les États et les particuliers sur le même plan; les particuliers ne sont pas sujets de droit international, et c'est exclusivement dans le domaine de ce droit que la Cour est appelée à fonctionner ». ${ }^{48}$ Si l'on remonte aux commissions instituées par les traités d'abolition de l'esclavage, considérées comme les ancêtres des mécanismes internationaux de protection des droits de l'homme, on constate que la procédure ne prévoyait pas d'accès direct de la victime de la violation et qu'aucun statut procédural n'était généralement accordé aux esclaves que l'on visait à protéger, fût-ce celui de témoin. ${ }^{49}$ Le contentieux devant les commissions mixtes instituées par des traités de paix, commerce et navigation, souvent présenté comme l'ancêtre de l'arbitrage d'investissement, est, quant à lui, fort bien résumé par une décision Dickson Carwheel de 1931 : celle-ci affirmait, avec un certain degré de généralité, que la relation de responsabilité internationale étant purement interétatique, il ne se crée aucun lien juridique à l'égard de l'individu, dès lors que celui-ci n'est point un sujet de droit international. ${ }^{50} \mathrm{Or}$, si la jurisprudence admet progressivement que l'individu peut bien être titulaire de droits et obligations internationales, son statut procédural reste fortement inhibé,

diante la regolare pubblicazione del trattato, identico il valore delle loro decisioni, appaiono come tribunali communi ai due Stati, come un organo unico vivente ad un tempo nelle due sfere giuridiche ».

47 G. Sperduti, L'individuo nel diritto internazionale (1950), 69. Pour une analyse de la pensée de l'auteur en français, faisant le lien avec les idées de sujet matériel et d'intérêt légitime, voir M. Frappier, L'individu, sujet matériel du droit international chez Giuseppe Sperduti, in IHEI, Les grandes pages du droit international Les sujets (2015), 227.

48 Comité consultatif des juristes, Procès-verbaux des séances du Comité (1920), 208.

49 J. S. Martinez, The Slave Trade and the Origins of International Human Rights Law (2012), 99 : " the voices of these individuals are curiously absent from the courts' proceedings. Only occasionally did they give testimony as witnesses. They were not directly represented in the trials. And while the slave trade is sometimes described as violating "human rights" in documents from the nineteenth century, the slaves themselves rarely appear in any legal proceedings as claimants of rights. Instead, they are silent bystanders-beneficiaries of the system, to be sure, but hardly active participants in it ».

50 Dickson Car Wheel Company (USA) c. États-Unis du Mexique, Commission États-Unis-Mexique, juillet 1931, IV RSA, 669. 
comme le démontre encore en 1950 la position du gouvernement français défendeur dans le différend Ottoz:

Il est exact que, à la différence des tribunaux arbitraux mixtes, la Commission de Conciliation ne peut trancher que des litiges entre Etats; mais les Gouvernements agissent dans l'intérêt - et pour assurer le respect des droits - des ressortissants de leurs pays; c'est surtout et presque exclusivement sur le plan de la procédure que le litige demeure strictement interétatique; sur le fond du droit, la Commission [...] est appelée à reconnaître ou à nier l'existence non pas seulement d'une obligation de l'État italien, mais d'un droit subjectif d'un ressortissant d'une des Nations Unies. ${ }^{51}$

Ce type d'argumentation n'est pas exempt d'un certain nombre d'incohérences, que Politis pointe très tôt du doigt : " on confond la valeur intrinsèque de ces règles avec leur mise en œuvre : si elles s'adressent aux États, c'est uniquement parce que dans l'état actuel de l'organisation internationale, leur réalisation ne peut pas se passer de leur intermédiaire; mais elles visent principalement et directement l'individu ${ }^{52}$ Quelques années plus tard, dans son cours de La Haye de 1962, von der Heydte dénonce que " considérer $[\ldots$.$] comme sujet de droit uniquement celui qui peut faire va-$ loir un droit soit en justice soit par l'emploi de la force » est un avis qui " confond les causes et les effets ".53 Dans un ouvrage de la même année, Nørgaard souligne que la posture traditionnelle revient à entretenir un amalgame entre la question de la possession de droits et obligations avec celle de la capacité procédurale à être demandeur ou défendeur en justice. $^{54}$

Quoi qu'il en soit, l'introduction d'une historicité conduit à percevoir le caractère parfois stérile de la réflexion classique sur le sujet. Cela ne veut pas dire que le contentieux international mixte, ayant explosé de nos jours, n'ait pas considérablement concouru au développement de la personnalité active des personnes privées en droit international. ${ }^{55}$ Il suffit notamment de penser à la récente sentence Urbaser, qui arrive à affirmer que :

51 Différend Ottoz, Commission de conciliation franco-italienne, décision $\mathrm{n}^{\circ} 85$ rendue le 18 septembre 1950, RSA, vol. XIII, pp. 232-242, p 236.

52 N. Politis, Les nouvelles tendances du droit international (1927), 7.

53 F. A. von der Heydte, L'individu et les tribunaux internationaux, t. 107 RCADI (1962), 307.

54 C. A. Nørgaard, The Position of the Individual in International Law (1962), 11.

55 A. Peters, Beyond Human Rights - The Legal Status of the Individual in International Law (2017). 
In light of this more recent development, it can no longer be admitted that companies operating internationally are immune from becoming subjects of international law. ${ }^{56}$

\section{Une généalogie idéologique différente}

Si on essaie de retracer l'évolution de la réflexion sur l'action individuelle, on constate que celle-ci surgit de deux manières différentes dans les deux champs étudiés. En effet, en droit international des investissements, il existait de longue date une pratique d'arbitrage commercial où l'État était parfois amené à intervenir comme défendeur face à une personne privée, dans le cas de contentieux portant sur des contrats d'État. ${ }^{57} \mathrm{La}$ réflexion doctrinale intervient en aval par rapport à ce phénomène qu'elle essaie de théoriser : la question qui se pose est celle de savoir si ces contrats sont ancrés dans l'ordre juridique international, ce qui a donné naissance au débat sur la Grundlegung. ${ }^{58}$ Ainsi, l'une des premières réflexions doctrinales en la matière, celle de Mann en 1944, reprend un corps consistant de sentences arbitrales rendues dès le début du XIX ${ }^{\text {ème }}$ siècle afin de systématiser une pratique déjà foisonnante d'arbitrage mixte. ${ }^{59}$

Au contraire, dans le cadre des droits de l'homme, la réflexion théorique a servi de terreau à l'émergence du recours individuel. Le tournant libéral de la doctrine internationaliste à partir de la fin du XIX ${ }^{\text {ème }}$ siècle avait déjà permis de théoriser l'élévation au niveau du droit international de certains droits de la personne humaine ${ }^{60}$ : ainsi, Fiore en $1890^{61}$ ou Mandelstam dans l'entre-deux-guerres ${ }^{62}$ parviennent à dresser une liste de six droits fondamentaux devant être internationalisés. Le recours individuel semble

56 Urbaser S.A. and Consorcio de Aguas Bilbao Bizkaia, Bilbao Biskaia Ur Partzuergoa c. République d'Argentine, affaire CIRDI n. ARB/07/26, sentence du 8 décembre 2016, $\$ 1195$.

57 Voir parmi les différents cours de La Haye sur le sujet, les remarquables analyses de Ch. Leben, La théorie du contrat d'Etat et l'évolution du droit international des investissements, t. 302 RCADI (2003), 212-263.

58 P. Mayer, Le mythe de l'ordre juridique de base' ou 'Grundlegung', in RIDC, Le droit des relations économiques internationales : études offertes à Berthold Goldman (1982), 199-216.

59 F. A. Mann, The Law Governing State Contracts, BYBIL (1944), 11-33.

60 M. Koskenniemi, The gentle civilizer of nations : the rise and fall of international law 1870-1960 (2001), 11-97, surtout 54.

61 P. Fiore, Le droit international codifié et sa sanction juridique (1890), 87-90.

62 A. Mandelstam, La protection internationale des droits de l'homme (1931). 
donc sceller et prolonger l'exigence d'une protection internationale des droits de la personne.

Jean Spiropoulos a été l'un des premiers à comparer les deux types de recours directs. ${ }^{63}$ En reprenant une bipartition assez répandue à l'époque, il distingue entre l'action de l'individu contre l'État étranger et l'action de l'individu contre son propre État. Cette summa divisio reflète déjà l'émergence d'un discours de divergence entre les deux mécanismes : le premier destiné à protéger un étranger dans le chef duquel le standard minimum de traitement était violé, le second voué à faire valoir au plan international le respect de certains droits fondamentaux dus par l'État à ses ressortissants (conception ayant largement évolué de nos jours). Spiropoulos distingue donc les deux cas de figure en raison de la nature différente de droits immédiats issus de l'ordre juridique international dans les deux cas et de la position différente de l'individu en question par rapport à la souveraineté de l'État.

Les intuitions de Spiropoulos sur l'évolution du contentieux trouvent d'ailleurs confirmation dans le foisonnement de la pratique juridictionnelle de la seconde moitié du XX ${ }^{\text {ème }}$ siècle, où l'individu-demandeur joue un rôle central. Cette évolution a été considérée comme un changement de paradigme, que l'on a pu résumer par l'idée d'humanisation du droit international. C'est ainsi que Maurice Bourquin intitule un célèbre article datant de la moitié du siècle, théorisant l'évolution d'un droit qui n'est plus enfermé dans les sphères de la haute politique, mais parle désormais un langage plus humain. ${ }^{64}$ C'est le début d'une vague idéologique qui, en réaction aux atrocités de la Seconde Guerre mondiale, prône l'émergence d'une volonté axiologique tendant à faire du droit international un instrument de protection de l'homme, caractérisant le passage du droit international classique au droit international contemporain. ${ }^{65}$ On assiste du moins à un changement de posture intellectuelle par rapport au modèle

63 J. Spiropoulos, L'individu et le droit international, t. 30 RCADI (1929), 192- 270. Voir également l'article d'A. K. Fortas, L'individu sujet de droit international selon Jean Spiropoulos, in IHEI, Les grandes pages du droit international - Les sujets (2015), 273.

64 M Bourquin, L'humanisation du droit des gens, in La technique et les principes du droit public : études en l'honneur de George Scelle (1950), 21-54.

65 R. Cassin, L'homme, sujet de droit international et la protection des droits de l'homme dans la société universelle, in La technique et les principes du droit public : études en l'honneur de George Scelle, t. 1 (1950), notamment 81-82; A.A. Cançado Trindade, International Law for Humankind : Towards a New Jus Gentium - General Course on Public International Law, 316 RCADI (2005), notamment 252-317. 
positiviste, l'homme est désormais placé au cœur du droit des gens aussi bien par la lecture sociologique de Scelle, ${ }^{66}$ que par la récupération du premier jusnaturalisme, notamment des ouvres de Vitoria et Grotius, dans les pages d'Antônio Augusto Cançado Trindade aujourd'hui. ${ }^{67}$

Le ius standi de l'individu est alors conçu comme le corollaire de ce processus d'humanisation, dès lors qu'il reflète l'idéal universalisant des droits de l'homme. ${ }^{68}$ Néanmoins, cette idée semble pouvoir décrire seulement une partie de la question et négliger l'existence de phénomènes collatéraux comme l'accès de l'investisseur aux tribunaux arbitraux, des fonctionnaires aux juridictions spécialisées ou de l'ouverture du Tribunal international du droit de la mer (TIDM) aux particuliers dans des cas résiduels. ${ }^{69} \mathrm{Il}$ convient par ailleurs de souligner que, en l'état actuel du contentieux international, la personne morale bénéficie également de possibilités de recours et que cette faculté n'est nullement exclusive de la personne humaine. ${ }^{70}$

66 G. Scelle, Précis de droit des gens (1932), 9, qui soutient que seul l'individu peut être sujet de l'ordre juridique, interne ou international : " si la qualité de sujet de droit n'appartient pas à tous les individus et ne leur appartient pas uniformément, elle ne peut cependant appartenir qu'à des individus. Elle est en effet un attribut social de la volonté ».

67 A. A. Cançado Trindade, The access of individuals to international justice (2011), 15.

68 P. Guggenheim, Les principes de droit international public, t. 80 RCADI (1952) 116, 121 : "seulement dans le cadre de la protection des droits de l'homme qu'on a cherché à donner à l'individu en droit international une capacité juridique immédiate dépassant la position fragmentaire ».

69 Pour un excellent panorama utilisant cette clef de conceptualisation, voir J. A. Pastor Ridruejo, La humanización del derecho internacional y el acceso del individuo a sus instituciones jurisdiccionales, in Y. Gamarra Chopo (dir.), Lecciones sobre justicia internacional (2009), 13-24.

70 Concernant de la faculté d'action de la personne morale, la question déborde du cadre de cette étude; voir D. Müller, La protection de l'actionnaire en droit international (2015). De manière impressionniste, nous pouvons rappeler que l'arbitrage d'investissement tend à n'établir aucune différence entre les droits procéduraux d'un investisseur personne privée ou personne morale. La raison idéologique réside dans le fait qu'on considère l'action dans une société locale en tant qu'investissement et qu'on ne fait point de différence entre actionnaires minoritaires et majoritaires (CMS Gas Transmission Company c. République d'Argentine, affaire CIRDI n ${ }^{\circ} \mathrm{ARB} / 01 / 8$, décision du comité ad hoc du 25 septembre 2007, \$73). Cela conduit le plus souvent à un phénomène problématique de multiplication des contentieux en tiroirs, des procédures parallèles et concurrentes par rapport à un même complexe factuel (E. Gaillard, Abuse of Process in International Arbitration, 32(1) ICSID Review (2017), 17-37.).

En revanche, dans le contentieux $\mathrm{CEDH}$, la prise en compte de la personnalité morale conduit à une distinction des rôles procéduraux : les actionnaires 
En revanche, la perspective constitutionnaliste va au-delà de cette notion d'humanisation. Anne Peters a notamment abordé cette évolution de manière bien plus transversale, en soulignant que la transformation du rôle de l'individu dans l'ordre juridique international intervient également " jenseits der Menschenrechte ", au-delà des droits de l'homme, dès lors que celui-ci tire de l'ordre juridique international toute une série de droits subjectifs qui débordent largement le périmètre de ceux-là. ${ }^{11} \mathrm{La}$ question sera donc de savoir si l'accès à la juridiction internationale peut être, dans les deux cas, qualifié de droit subjectif de l'individu ou si une nuance peut être introduite.

\section{B. Une diversité sous-jacente}

Lévolution du discours sur l'accès de l'individu à la juridiction internationale fait émerger l'existence d'une différence d'optique entre les deux champs analysés, différence qui se retrouve dans les travaux préparatoires des deux principaux instruments aménageant aujourd'hui ce type de recours (1). Cela ne saurait étonner, dès lors que la nature du recours reflète une configuration différente de la position de l'individu par rapport à l'action de l'État souverain (2).

n'agissent individuellement que pour défendre leurs intérêts propres, qui ne sauraient être revendiqués par la société. La Cour affirme clairement dans Agrotexim que « la Cour n'estime justifié de lever le "voile social" ou de faire abstraction de la personnalité juridique d'une société que dans des circonstances exceptionnelles, notamment lorsqu'il est clairement établi que celle-ci se trouve dans l'impossibilité de saisir par l'intermédiaire de ses organes statutaires ou - en cas de liquidation - par ses liquidateurs les organes de la Convention. La jurisprudence des cours suprêmes de certains Etats membres du Conseil de l'Europe va dans le même sens. La Cour internationale de Justice a également consacré ce principe en ce qui concerne la protection diplomatique de sociétés (arrêt Barcelona Traction, Light and Power Company Limited, du 5 février 1970, Rec. CIJ 1970, avis consultatifs et ordonnances 1970, pp. 39 et 41, paras. 56-58 et 66) » (CEDH Agrotexim c. Grèce, arrêt du 24 octobre 1995, requête n 14807/89, \$66).

71 A. Peters, Jenseits der Menschenrechte : Die Rechtsstellung des Individuums im Völkerrecht (2014), 469. 


\section{Une diversité résultant des travaux préparatoires}

Témoins de l'évolution idéologique qui a conduit à l'insertion d'un mécanisme d'accès direct de l'individu à la juridiction dans les engagements conventionnels, les travaux préparatoires de la CEDH et de la Convention CIRDI, les deux instruments qui ont le plus contribué à cette évolution à ce jour, révèlent deux logiques distinctes ayant motivé depuis le début une différence d'approche.

Les minutes de la Conférence des Hauts Fonctionnaires, réunis sous l'égide du Conseil de l'Europe en juin 1950, attestent que la naissance du recours individuel au sein du contentieux européen des droits de l'homme tient à une proposition norvégienne tendant à l' " ouverture d'une voie de recours à l'individu $\gg .^{72}$ Cette proposition sera immédiatement accueillie avec enthousiasme par le représentant français, M. Chaumont, qui tentera d'en motiver le bien-fondé en rappelant qu'il est un "principe général du droit que l'action en justice suit nécessairement le droit reconnu $» .{ }^{73}$ Dans ces mots transparaît l'influence de la théorie subjectiviste héritée de Savigny et qui voit dans l'action le simple prolongement du droit subjectif de l'individu.

Cette vision subjectiviste du recours individuel va se répercuter sur la jurisprudence de la Commission des droits de l'homme. En effet, dans une décision De Vos c. Belgique de 1958, celle-ci semble avoir adhéré aux raisons idéologiques alléguées au sein de la conférence des parties pour interpréter la nature de l'article 25 de l'époque. De son avis, «le droit de recours à la Commission figure parmi [1]es droits et libertés » reconnus dans la Convention. ${ }^{74} \mathrm{La}$ Commission ne va pas jusqu'à affirmer que l'article 25 incarne simplement les droits garantis par la Convention mis en mouvement, mais présente le recours individuel comme ayant la consistance d'un véritable droit individuel, protégé par la Convention au même titre que les autres droits de l'homme, en restant attachée à une lecture tendanciellement subjectiviste de l'action. Cette décision ne fait pas figure de cas isolé. La Commission a développé toute une jurisprudence concernant les cas d'États empêchant la communication du requérant avec elle. Dans ces cas, la Commission semble admettre que, si l'individu peut démontrer que

72 Conseil de l'Europe, Nature juridique de l'obligation découlant de l'article 25 a) in fine de la Convention - Note du Secrétariat, A86.480, 2 : «Dans le compte rendu de la séance du 10 juin 1950 [...], M. Sund (Norvège) se déclare partisan de l'ouverture d'une voie de recours à l'individu ».

73 Ibid.

74 CommEDH, De Vos c. Belgique, décision du 20 mars 1958, requête n²19/56. 
cette atteinte lui a causé un préjudice matériel ou moral, une violation d'un droit au recours individuel est envisageable. ${ }^{75}$ On ne saurait néanmoins lire ce contentieux de manière exclusivement subjective, puisque la Commission a clairement énoncé que le droit de recours constitue également le pilier de "l'ordre public européen $»{ }^{76}$ réintroduisant donc une dimension objective.

Une optique différente ressort des travaux préparatoires de la Convention de Washington. La question de l'accès direct de la personne privée et des entreprises est présentée par Aron Broches, président du Comité consultatif des experts juridiques chargés de la rédaction de la Convention, comme la raison centrale de l'exigence d'un tel instrument conventionnel. ${ }^{77}$ Cela est cristallisé dans le préambule duquel on déduit l'importance de l'arbitrage mixte aux fins de la dépolitisation des litiges liés à l'investissement. La préférence pour un règlement pacifique mixte l'emporte sur les motivations liées aux droits subjectifs des investisseurs.

Au sein du Comité, il est question d'un " novel right of direct access $»^{78}$ de la personne privée à un forum international. Le président Broches resitue ce débat dans la continuité de la reconnaissance progressive de l'individu en tant que sujet de droit international, ${ }^{79}$ en adoptant une perspective différente du Comité des dix. Le représentant chilien inscrit la Convention dans la lignée des affirmations des internationalistes qui avaient longtemps proclamé que l'individu était porteur de droits et obligations également sur le plan international. Néanmoins, il déclare également, de manière très significative, que la Banque mondiale transposait au plan international certaines initiatives comparables existant préalablement seulement au niveau régional, c'est-à-dire celle de la Cour de justice centre-américaine et de la Cour de justice des Communautés européennes ${ }^{80}$ Le choix de ne pas se référer aux juridictions protégeant les droits de l'homme ne tient certainement pas au hasard.

75 CommEDH, Ganthaler c. RFA, décision du 10 janvier 1959, requête n³63/58.

76 CommEDH, Autriche c. Italie, décision sur la recevabilité du 11 janvier 1961, requête $n^{\circ} 788 / 60$.

77 Intervention du président A. Broches au sein du Comité consultatif des experts juridiques, séance du 29 avril 1964, in History of the ICSID Convention (1968), vol. II(1), 495 .

78 Intervention de M. Mankoubi (Togo) au sein du Comité consultatif des experts juridiques, séance du 20 décembre 1963, in History of the ICSID Convention (1968), vol. II(1), 293.

79 Intervention du président A. Broches au sein du Comité consultatif des experts juridiques, séance du 3 février 1964, in History of the ICSID Convention (1968), vol. II(1), 303. 
De manière générale, la justification du nouveau mécanisme procédural réside dans des considérations pragmatiques qui sont bien plus présentes que la logique de droits subjectifs appartenant à l'investisseur. Il est question d'accorder une faculté nouvelle à l'investisseur, lui permettant de s'émanciper des dysfonctionnements et des lacunes du règlement interétatique des différends, et non pas de lui reconnaître un droit découlant naturellement de la protection internationale. Aux objections de ceux qui préferent un mécanisme de règlement des différends interétatique, Broches répondait en effet que :

$[\mathrm{T}]$ his progressive development of international law might be especially valuable in cases where smaller States had to deal with investors from larger countries, and the likelihood of successful inter-State negotiations was somewhat reduced. The Convention proceeded on the assumption that, in the stated situations, States would be willing to have direct dealings with investors. ${ }^{81}$

Il est davantage question ici d'une volonté des États d'accorder un mécanisme procédural que de reconnaitre un droit fondamental de l'individu. Ce mécanisme répondrait, en effet, à des nécessités de rééquilibrage des asymétries de puissance économique existant dans la communauté internationale et non pas à celui d'humanisation qui se reflète dans la Convention de sauvegarde. De plus, la Convention de Washington ne contient aucun droit substantiel, alors que la Convention européenne de sauvegarde des droits de l'homme et des libertés fondamentales est dédiée à leur proclamation. Le glissement de la lecture du droit de recours du mécanisme procédural vers un droit de l'individu est facilité par ce contexte.

\section{Une diversité dépendant de la relation de l'individu à l'État}

L'optique de l'histoire des idées adoptée suggère également un parallèle entre l'émergence de l'accès direct de l'individu à la juridiction internationale et la formation du recours de droit public de l'individu dans l'ordre interne.

80 Intervention de M. Brunner (Chili) au sein du Comité consultatif des experts juridiques, séance du 12 juin 1964, in History of the ICSID Convention (1968), vol. II(1), 305 .

81 Intervention du président A. Broches au sein du Comité consultatif des experts juridiques, séance du 28 avril 1964, in History of the ICSID Convention (1968), vol. II(1), 495 . 
En premier lieu, il a été démontré que le recours administratif est le résultat d'une évolution politico-idéologique propre au XIX ${ }^{\text {ème }}$ siècle qui ne voit plus dans l'individu un simple objet de la puissance publique, mais progressivement le détenteur de droits publics subjectifs. ${ }^{82}$ Après une première moitié du siècle durant laquelle cette idée originaire a été déconstruite, c'est lors de la seconde moitié que surgit une réflexion sur l'architecture juridique du recours à proprement parler. En droit international, le débat intellectuel sur le recours est décalé d'un siècle : c'est lors de la première moitié du XXème siècle que l'on situe la pars destruens et pendant sa deuxième que débute une pars costruens, non encore achevée. Néanmoins, une communauté idéologique de base est frappante : cette première idée fait remarquablement écho à la conclusion du cours de La Haye de Jean Spiropoulos, ${ }^{83}$ prônant la remise en cause de l'ancienne vision hégélienne de la souveraineté 'absolue' de l'État et la prise de conscience de l'existence de droits subjectifs immédiats que l'individu tient de l'ordre juridique international.

L'accès direct de l'individu à la juridiction internationale ne peut être pensé que si l'on se détache de la vision de l'État propre au positivisme classique. La doctrine essaie de tirer les conséquences de cette évolution de différentes manières. D'un côté, dans l'optique du constitutionnalisme global, l'individu est devenu un véritable sujet du droit international et titulaire de droits subjectifs d'origine internationale. ${ }^{84}$ De l'autre, l'on peut situer le rejet même de la dichotomie objet-sujet comme non-pertinente : c'est ce que fait l'École de New Haven qui préfere voir dans la personne privée un "participant» au processus décisionnel du droit international et souligner que la rareté de l'accès direct de celui-ci aux juridictions internationales n'était donc pas due à la nature du droit international. ${ }^{85}$ En tout état de cause, l'individu est à tout le moins devenu un " usager " certain du droit international et n'est plus totalement asservi à l'État souverain. ${ }^{86}$

82 A. Gaillet, L'individu contre l'État; essai sur l'évolution des recours de droit public dans l'Allemagne du XIXe siècle (2012), 26.

83 Spiropoulos, supra note 63, 265.

84 A. Peters, The Subjective International Right, 59 Jahrbuch des öffentlichen Rechts der Gegenwart (2011), 411-456.

85 R. Higgins, Conceptual Thinking about the Individual in International Law, 4(1) British Journal of International Studies (1978), 1-19.

86 E. Roucounas, The Users of International Law, in M. H. Arsanjani et al. (dir.), Looking to the Future : Essays on International Law in Honor of M.W Reisman (2010), 217-234. 
En second lieu, la comparaison des recours de droit public montre que la différence entre eux dépend de la relation entre la structure du recours et la relation de l'individu face à l'État. ${ }^{87}$ Ainsi, la différence entre le recours de l'investisseur et le recours de la personne en matière de droits de l'homme peut être théorisée en raison de la position différente qu'a l'individu face à l'État dans les deux cas. Opérateur économique dans le premier, revendiquant des droits de traitement accordés par la puissance souveraine qui a admis l'investissement sur son territoire, il s'agit dans le second d'une personne titulaire de droits humains en raison de sa simple soumission à l'autorité de l'État et de sa nature même. ${ }^{88}$ C'est également ce que semble affirmer le juge Cançado Trindade dans l'une de ses opinions dissidentes :

The juridical capacity varies in virtue of the juridical condition of each one to undertake certain acts. Yet although such capacity of exercise varies, all individuals are endowed with juridical personality. Human rights reinforce the universal attribute of the human person, given that to all human beings correspond likewise the juridical personality. ${ }^{89}$

\section{La perspective synchronique}

Les discours des juridictions étudiées révèlent une analyse hétérogène de la nature de l'accès direct à la juridiction internationale. Le vocabulaire des juges permet de constater une différence de posture dans les deux cadres (A). Ce décalage s'explique et se reflète surtout dans le contentieux préliminaire, lieu de formation du lien d'instance où se cristallise donc la compréhension que le juge se fait du principe de justice consensuelle (B).

87 A. Gaillet, Der Einzelne gegen den Staat. Die Geschichte der Rechtsbehelfe des öffentlichen Rechts in Deutschland und Frankreich im 19. Jahrhundert, 129(1) Zeitschrift der Savigny-Stiftung für Rechtsgeschichte. Germanistische Abteilung (2012), 109-149, 111, qui parle d'une «Zusammenhang zwischen der Struktur der Rechtsbehelfe und dem Grundverhältnis zwischen dem Einzelnen und dem Staat ».

88 H. Ruiz Fabri, Droits de l'homme et souveraineté de l'État : les frontières ont-elles été substantiellement redéfinies?, in Mélanges Fromont, Les droits individuels et le juge en Europe (2001), 371.

89 Juridical Condition and Human Rights of the Child, CIADH avis consultatif OC-17/2002 du 28 août 2002, opinion dissidente d'A. A. Cançado Trindade, $\mathbb{} 34$. 


\section{A. Un contentieux révélateur de la nature du recours}

La lecture des discours juridictionnels permet de mettre en évidence un décalage entre les vocabulaires employés par les deux juridictions. Si la jurisprudence de la $\mathrm{CEDH}$ a façonné un véritable «droit fondamental » au recours individuel (1), ${ }^{90}$ la pratique arbitrale reste divisée quant à la qualification de l'accès à l'arbitrage comme droit de l'investisseur (2).

\section{Un droit fondamental au recours devant la juridiction européenne}

Déjà dans les travaux du Secrétariat de la Commission sur la nature de l'obligation insérée à l'article 25 de la $\mathrm{CEDH}$, trois options différentes concernant la nature juridique du droit de recours individuel étaient distinguées : dans une première perspective, le droit de recours serait un droit individuel et subjectif reconnu par la Convention, comparable à ceux du titre premier ; dans une perspective opposée, aucun droit individuel ne correspond à l'obligation de l'État de prévoir un recours individuel, qui implique simplement un lien procédural entre la Commission et l'État défendeur ; dans une optique intermédiaire, il s'agirait d'un droit individuel sui generis, de nature procédurale et non matérielle, projetant sur le plan du contentieux international les droits protégés par la Convention. ${ }^{91}$

Si la jurisprudence de la Commission semblait pencher vers la première branche, la Cour européenne des droits de l'homme opte davantage pour la troisième conception dans sa jurisprudence relative à l'article 34 de la Convention. C'est notamment la jurisprudence en matière d'extradition et respect des mesures conservatoires relatives à la personne réclamée qui a offert à la Cour l'occasion d'identifier la nature et la consistance du recours individuel. Dans son arrêt Cruz Varas de 1991, elle affirme déjà que le recours individuel est un "droit de nature procédurale, à distinguer des droits matériels énumérés au titre I de la Convention ", qui est soumis à l'adage Airey selon lequel la Convention doit s'interpréter comme garantis-

90 E. Lambert-Abdelgawad, La saisine de la Cour européenne des droits de l'homme, in H. Ruiz Fabri, J.-M. Sorel La saisine des juridictions internationales (2006), 212.

91 Conseil de l'Europe, Nature juridique de l'obligation découlant de l'article 25 a) in fine de la Convention - Note du secrétariat, A86.480, 49-56. 
sant des droits concrets et effectifs et non pas théoriques et illusoires. ${ }^{92}$ C'est surtout l'arrêt Mamatkoulov et Askarov qui, tout en abandonnant la position de 1991 concernant le caractère non obligatoire des mesures conservatoires, va donner des précisions fondamentales concernant la texture normative du droit au recours individuel. ${ }^{93} \mathrm{La}$ Cour y précise que celui-ci est «l'un des piliers essentiels de l'efficacité du système de la Convention »; il en est une " disposition clé » qui participe du " caractère singulier de la Convention » en tant que traité de garantie collective des droits de l'homme, et donc aussi de l'exigence d'effectivité de ses dispositions.

Cette analyse se pérennise au fil de la jurisprudence, qui acte le retour de certaines idées fondamentales : le droit de recours individuel est un droit de nature procédurale, relevant du noyau dur des droits protégés par la Convention, dont l'exigence d'effectivité est centrale aux fins de la garantie du système conventionnel, notamment depuis son évolution avec le protocole $\mathrm{n}^{\circ} 11 .{ }^{94}$ Ainsi, il ne semble pas exagéré d'affirmer qu'une telle jurisprudence a reconnu un véritable droit d'action individuelle qui devient, grâce à la configuration post protocole $\mathrm{n}^{\circ} 11$, un « droit inconditionnel d'accès direct à la Cour européenne ${ }^{95}$

\section{Le débat sur la titularité des droits et les contre-mesures touchant l'investisseur}

Cette analyse n'est pas transposable au cas de l'arbitrage d'investissement. Dans ce cadre, c'est le contentieux portant sur les contre-mesures qui a donné lieu à des réflexions prétoriennes sur la nature des droits de l'inves-

92 Cruz Varas et autres c. Suède, CEDH arrêt du 20 mars 1991, Requête no 15576/89, \99: «Il confere de la sorte au requérant un droit de nature procédurale, à distinguer des droits matériels énumérés au titre I de la Convention et dans les protocoles additionnels. Il résulte toutefois de l'essence même de ce droit que les particuliers doivent pouvoir se plaindre de sa méconnaissance aux organes de la Convention. A cet égard aussi, la Convention doit s'interpréter comme garantissant des droits concrets et effectifs, et non théoriques et illusoires (arrêt Soering précité, série A no 161, p. 34, \$87, avec les références) ».

93 Mamatkoulov et Askarov c. Turquie, CEDH arrêt du 4 février 2005, Requêtes nos $46827 / 99$ et $46951 / 99, \mathbb{1} 100-102$.

94 Chamaïev c. Géorgie et Russie, CEDH arrêt du 12 avril 2005, Requête $\mathrm{n}^{\circ}$ 36378/02, \470-472; Aoulmi c. France, arrêt du 12 janvier 2006, Requête $n^{\circ}$ 50278/99, \103-107.

95 S. Bartole, B. Conforti et G. Raimondi, Commentario alla Convenzione europea per la tutela dei diritti dell'uomo e delle libertà fondamentali (2001), 626-627. 
tisseur. En effet, dans le cadre de l'ALENA, plusieurs investisseurs ont attaqué le Mexique en raison de mesures fiscales considérées discriminatoires. Selon la défense mexicaine, les faits contestés n'étaient autres que des contre-mesures licites, adoptées en réponse aux actes illicites commis par l'État de nationalité de l'investisseur. Pour évaluer la validité de l'argument, le tribunal arbitral devait se prononcer sur la titularité des droits découlant du traité bilatéral d'investissement (TBI) : en prenant ses contre-mesures, le Mexique avait-il affecté les droits de l'État auteur présumé de l'illicite ou bien les droits d'un tiers détenteur de ces droits, c'est-à-dire de l'investisseur? Cette saga a donné lieu à une réflexion plus générale sur la typologie des droits en cause, qui a divisé la jurisprudence et la doctrine. ${ }^{96}$

Trois postures peuvent être distinguées. La première, dite théorie des droits dérivés, identifie dans les différents droits de l'investisseur des droits purement interétatiques : l'investisseur acquiert un droit de réclamation qui n'est en réalité qu'une faculté procédurale lui permettant de se substituer à l'État, "stepping into the shoes ${ }^{97}$ de celui-ci. La sentence Loewen avait déjà ébauché l'idée selon laquelle les investisseurs seraient simplement « permitted for convenience to enforce what are in origin the rights of Party states $»{ }^{98}$ Il s'agirait ici d'une sorte de renforcement de la protection diplomatique ${ }^{99}$ ou d'une protection diplomatique inversée impliquant un endossement des droits de l'État. ${ }^{100}$ Telle semble être la position du Conseil d'État français selon qui un TBI « ne crée d'obligations qu'entre

96 N. Iwatsuki, '国籍国に対する対抗措置としての正当性と投資家への対抗可能 性' 14-J-008 RIETI Discussion Paper Series (2014). L'auteur resitue dans une perspective historique la question de la pertinence de l'argument des contre-mesures en tant qu'élément de défense pour l'État ayant violé le droit de propriété des étrangers investissant sur son territoire. Il suggère qu'au cœur du débat se trouve une question d'interprétation du TBI : est-ce qu'on considère la relation investisseur-État comme complètement déconnectée de celle interétatique?

97 Archer Daniels Midland Company and Tate \& Lyle Ingredients Americas, Inc. c. États-Unis du Mexique, affaire CIRDI n ARB (AF)/04/5, sentence du 21 novembre 20007, $\mathbb{1 6 3 .}$

98 Loewen Group, Inc. and Raymond L. Loewen c. États-Unis, affaire CIRDI $\mathrm{n}^{\circ}$ $\mathrm{ARB}(\mathrm{AF}) / 98 / 3$, sentence du 26 juin 2003, $\mathbb{} 233$.

99 J. Crawford, ILC's Articles on Responsibility of States for Internationally Wrongful Acts : A Retrospect, 96 AJIL (2002), 874, 888.

100 M. Forteau, La contribution au développement du droit international général de la jurisprudence arbitrale relative aux investissements étrangers, IV(1) Anuário Brasileiro de Direito Internacional (2009), 38 : "sorte de protection diplomatique inversée qui conduit à voir dans l'action contentieuse de l'investisseur étranger un endossement des droits de l'État». 
les deux États signataires ", ${ }^{101}$ ainsi que celle de la Cour constitutionnelle allemande. ${ }^{102} \mathrm{La}$ position intermédiaire, définie parfois comme théorie des droits contingents, a été consacrée par la sentence Archer Daniels. Celle-ci affirme que les investisseurs sont doués de droits procéduraux leur permettant de faire valoir de manière indirecte des droits substantiels qui restent dans la sphère interétatique. ${ }^{103}$ Ainsi, par une analogie contractuelle, ils sont des " third parties beneficiairies of the treaties ». ${ }^{104}$ En revanche, la théorie des droits directs prend le pari de comprendre les droits de l'investisseur comme lui étant propres et directement issus des instruments de protection. C'est la position défendue dans les sentences Corn et Cargill, et confirmée par les juridictions anglaises. ${ }^{105}$ La première affirme clairement

101 Conseil d'État, arrêt du 21 décembre 2007, n²80264 (inédit au recueil Lebon) : «Considérant que les stipulations de l'article 3 de l'accord entre le gouvernement de la République française et le gouvernement de la République algérienne démocratique et populaire sur l'encouragement et la protection réciproques des investissements, signé à Alger le 13 février 1993 ne crée d'obligations qu'entre les deux États signataires; que M. A ne peut donc utilement s'en prévaloir à l'appui de ses conclusions dirigées contre la décision lui refusant un visa d'entrée en France ».

102 Bundesverfassungsgericht, Beschluss des Zweiten Senats vom 8. Mai 2007, 2 BvM 1/03 - Rn. (1-95), $\ 51$ (dans la version allemande, $\mathbb{5} 54$ dans la version anglaise) : "From an international-law point of view, the specific feature of the arbitration of disputes before the International Centre for Settlement of Investment Disputes is that private individuals are able to complain as claimants of the violation of an international agreement concluded between states. In terms of content, therefore, the violation of an obligation is complained of which is owed not directly to the private applicant, but to his or her home state, although the protective purpose of the agreement targets the interests of private investors. Rights and obligations of the opposing state emerge in such case constellations from an international agreement which as a rule contain a separate necessity clause; thus, such rights and obligations emerge from a relationship governed by international law ».

103 Archer Daniels Midland Company, supra note 97, $\$ 163-166,173$.

104 A. Bjorklund, Private Rights and Public International Law : Why Competition Among International Economic Law Tribunals Is Not Working, 59(2) Hastings Law Journal (2007-8), notamment 265.

105 Occidental Exploration and Production Company v. Ecuador, Court of Appeal arrêt du 9 septembre 2005 (2005), EWCA Civ. 1116, \$19-22 : " treaties may in modern international law give rise to direct rights in favor of individuals acting on their own behalf and without their national state's involvement or even consent». 
que l'investisseur détient des « rights of its own »106 et la deuxième clarifie que « investor possesses not only procedural rights of access, but also substantive rights ». ${ }^{107}$

Ce débat appelle deux sortes de commentaires. Premièrement, il est parlant que la même querelle existe en droit international pénal où il demeure débattu si la coutume internationale prohibant les crimes contre l'humanité s'impose à un niveau interétatique et ne fait que rejaillir sur l'indivi$\mathrm{du},{ }^{108}$ ou bien si elle aboutit à imposer des obligations internationales directement dans le chef des individus. ${ }^{109}$ Le débat sur la titularité de l'immunité des hauts fonctionnaires de l'État en matière pénale a une configuration similaire. ${ }^{110}$ Ainsi, l'on voit que la matrice intellectuelle de la subjectivité de l'individu continue de poser des problèmes bien concrets.

Deuxièmement, s'il n'est pas ici utile de prendre position sur la titularité des droits substantiels, il est nécessaire de souligner la différence conceptuelle dans le maniement du recours individuel dans les deux jurisprudences. L'existence même du débat dans le contentieux arbitral montre que l'on ne saurait inconsidérément qualifier l'accès direct de l'investisseur de droit subjectif, ou encore moins établir une quelconque équivalence entre les droits substantiels de protection de l'investisseur et sa faculté procédurale d'agir devant le tribunal arbitral. Les partisans du modèle des droits directs ont souvent une compréhension des droits issus des TBI comme relevant de la catégorie des droits de l'homme, de sorte qu'ils établissent un parallèle entre la nature du régime juridique mis en place par les TBI et les traités de protection des droits de l'homme, presque par équivalence normative entre les deux instruments. Or, ce rapprochement peut faire l'objet

106 Corn Products International, Inc. c. États-Unis du Mexique, affaire CIRDI n ${ }^{\circ}$ ARB (AF)/04/1, sentence du 18 août 2009, $\mathbb{1} 174-176$. Dans le $\mathbb{1} 173$, le tribunal rappelle qu'il faut abandonner la fiction Mavrommatis quand on lit l'arbitrage d'investissement, "there is no need to continue that fiction in a case in which the individual is vested with the rights to bring claims of its own ».

107 Cargill, Incorporated c. États-Unis du Mexique, affaire CIRDI n ARB(AF)/05/2, 18 septembre 2009 , $\$ 423$. Au $\$ 426$, le tribunal précise qu'il ne faut pas confondre l'origine interétatique des droits avec les " holders of those rights ». Par rapport au droit d'accès direct, le tribunal prône l'abandon de la fiction Mavrommatis en citant explicitement la Cour d'appel britannique ( $\$ 386)$.

108 R. Maison, La responsabilité individuelle pour crime d'État en droit international public (2004).

109 B. Bonafé, The Relationship between State and Individual Responsibility for International Crimes (2009).

110 Pour un tableau du débat doctrinal, voir R. Pisillo Mazzeschi, The functional immunity of State officials from foreign jurisdiction : A critique of the traditional theories, Questions of International Law (2015). 
d'une critique fondamentale, dès lors que la nature des obligations étatiques est foncièrement différente : dans le cas des traités de protection des droits de l'homme, il s'agit d'obligations intégrales qui protègent des intérêts extraétatiques, alors que, dans le cadre des TBI, la réciprocité reste au cœur de l'instrument conventionnel dont la nature des obligations demeure synallagmatique. ${ }^{111}$

En ce qui concerne la nature du mécanisme d'accès direct, force est de constater que la Cour européenne des droits de l'homme a bien affirmé que le droit au recours individuel participe de la philosophie générale de la Convention comme instrument de protection des droits de l'homme. Dans l'arbitrage d'investissement, la logique normative étant différente, le mécanisme d'accès direct ne peut être lu à la même lumière d'un droit fondamental d'accès à la juridiction internationale. Cette différence se reflète dans les deux contentieux.

\section{B. Un contentieux préliminaire portant l'empreinte de la nature du recours}

Sans prétendre à l'exhaustivité, cette dernière partie veut démontrer que la nature procédurale différente du mécanisme d'accès à la juridiction se reflète également dans l'appréhension prétorienne de toute une série de questions contentieuses. La saisine n'est pas une question de compétence, dès lors qu'il est nécessaire de distinguer nettement consentement à la compétence et consentement à la saisine; néanmoins, elle possède un certain lien avec l'analyse des questions de compétence qui influencent le traitement du contentieux préliminaire. ${ }^{112}$

La divergence des discours dans ce cadre s'explique à deux égards. D'une part, le contraste tient à une conception différente de la place de l'État par

111 A. Gourgourinis, Investors' Rights Qua Human Rights? Revisiting The 'Direct'/'Derivate' Rights Debate, in M. Fitzmaurice (dir.), The Interpretation and Application of the European Convention of Human Rights (2012), 147-182. Lauteur critique ouvertement la position de Zachary Douglas, The International Law of Investment Claims (2009), 94, qui établit une équivalence normative fort contestable entre tous les « international treaties that confer rights directly upon non-state actors, such as the European Convention on Human Rights, the Algiers Accords establishing the Iran/US Claims Tribunal, bilateral investment treaties, NAFTA, the Energy Charter Treaty, ASEAN and the ICSID Convention ".

112 M. Forteau, La saisine des juridictions internationales à vocation universelle (CIJ et TIDM), in H. Ruiz Fabri, J.-M. Sorel, La saisine des juridictions internationales (2006), 45-52. 
rapport au mécanisme de saisine dans le cadre de la CEDH et de l'arbitrage d'investissement (1). D'autre part, l'origine de la protection internationale est conçue de manière profondément différente par les juridictions en matière de droits de l'homme et de droit de l'investissement étranger (2).

\section{Deux analyses différentes de la place de l'État}

L'une des différences les plus frappantes qui se dégagent des deux raisonnements prétoriens relativement à la nature de l'accès direct est liée au rapport qu'entretient l'État défendeur avec celui-ci. Dans le cadre de la CEDH, la Cour a en effet progressivement dégagé de l'article 34 de la Convention toute une série d'obligations, l'État ne pouvant « entraver par aucune mesure l'exercice efficace de ce droit ». La logique de l'existence d'un véritable droit à la saisine du requérant est ici très forte, la Cour ayant clairement affirmé que la finalité de cette règle est de " garantir l'effectivité du droit de recours individuel ». ${ }^{113}$ Premièrement, la Cour a progressivement fait découler de cette disposition non pas seulement des obligations négatives, d'abstention de tout comportement tendant à rendre l'exercice du droit au recours plus complexe, mais également des obligations positives qui vont dans le sens d'un renforcement de la coopération de l'État avec la Cour en fournissant tous les éléments probatoires nécessaires à une analyse de l'affaire. ${ }^{114} \mathrm{Il}$ n'est pas anodin, notamment dans une optique comparative, de souligner qu'il existe bien des cas de violation de l'article 34 eo ipso. ${ }^{115}$

113 Paladi c. Moldavie, CEDH arrêt du 10 mars 2009, Requête n³9806/05, \$ 87. Voir aussi Sindicatul «Păstorul Cel Bun »C. Roumanie, CEDH arrêt du 9 juillet 2013, Requête $n^{\circ} 2330 / 09, \$ 79$ parlant d'une « obligation de garantir l'effectivité du droit de recours individuel ».

114 Bazorkina c. Russie, CEDH arrêt du 27 juillet 2006, Requête n69481/01, $\mathbb{\$} 170$ : "The Court reiterates that proceedings in certain type of applications do not in all cases lend themselves to a rigorous application of the principle whereby a person who alleges something must prove that allegation, and that it is of the utmost importance for the effective operation of the system of individual petition instituted under Article 34 of the Convention that States should furnish all necessary facilities to make possible a proper and effective examination of applications ». La Cour renforce ce principe en lisant l'article 34 comme une lex specialis, à comprendre également à la lumière de l'article $38(\$ 175)$.

115 Ex multis : Lambor c. Roumanie ( ${ }^{\circ} 1$ ), CEDH arrêt du 24 juin 2008, Requête $n$ ${ }^{\circ} 64536 / 01, \$ 217$ : «En ce qui concerne enfin les allégations du requérant relatives aux pressions auxquelles l'auraient soumis deux médecins militaires qui exerçaient leurs fonctions à la prison de Timişoara, la Cour considère qu'on peut $\mathrm{y}$ voir des actes d'intimidation, qui, combinés avec la non-communication au re- 
Deuxièmement, la jurisprudence de la Commission et de la Cour a progressivement déduit du caractère fondamental de ce droit de recours le fait que les réserves à l'article 25 (puis 34) de la Convention n'étaient pas «autorisées par cet article », ${ }^{116}$ dès lors qu'elles sont incompatibles « avec l'objet et le but du système » ${ }^{117}$ conventionnel. L'apport de la jurisprudence Belilos et Loizidou va dans le sens d'une confirmation de l'opinion dissidente d'Antônio Augusto Cançado Trindade dans l'affaire Castillo Petruzzi, selon laquelle le droit au recours individuel est perçu par les juridictions de protection des droits de l'homme comme la pierre angulaire du système conventionnel. ${ }^{118}$

En revanche, dans l'arbitrage d'investissement, la logique du droit au recours doit être rejetée. Cela tient à la lecture de la condition de compétence ratione voluntatis du tribunal arbitral. ${ }^{119}$ Comme a pu le souligner Brigitte Stern,

[I]t is of the utmost importance not to forget that no participant in the international community, be it a State, an international organization, a physical or legal person, has an inherent right of access to a jurisdictional recourse. ${ }^{120}$

quérant des documents dont il avait besoin pour étayer sa requête devant la Cour s'analysent en une entrave au droit de recours individuel garanti par l'article 34 de la Convention. Cette conclusion s'impose d'autant plus que le requérant, qui était enfermé dans un espace clos et avait, de ce fait, peu de contacts avec ses proches ou avec le monde extérieur, se trouvait dans une situation particulièrement vulnérable ».

116 Belilos c. Suisse, CEDH arrêt du 24 avril 1988, série A, n¹32, $\mathbb{4 2 .}$

117 Loizidou c. Turquie, CEDH arrêt du 23 mars 1995, Requête n¹5318/89, $\$ 75$.

118 Castillo Petruzzi and others v. Peru, CIADH décision sur les exceptions préliminaires du 4 septembre 1998 , opinion dissidente, $\mathbb{} 35$ ( $"$ the right of individual petition is undoubtedly the most luminous star in the universe of human rights »). Voir aussi A. A. Cançado Trindade, Las cláusulas pétreas de la protección internacional del ser humano : El acceso directo de los individuos a la justicia a nivel internacional y la intangibilidad de la jurisdicción obligatoria de los tribunales internacionales de derechos humanos, 1 El Sistema Interamericano de Protección de los Derechos Humanos en el Umbral del Siglo XXI-Memoria del Seminario (1999), 3-68.

119 Forteau, supra note 112, 11 : «Le principe du consentement à la juridiction fait obstacle en effet au développement au sein de ce dernier [le contentieux interétatique] d'un droit de saisir le juge, y compris en cas d'atteinte à des normes fondamentales".

120 Impregilo S.p.A. c. République d'Argentine, affaire CIRDI n ARB/07/17, opinion dissidente de B. Stern du 21 juin 2011, $\mathbb{} 53$. 
Cette particularité du contentieux international a animé une certaine critique de l'arbitrage d'investissement, critique qui implique néanmoins une méconnaissance du rôle du consentement de l'État. Si la pratique est allée vers un élargissement considérable des conditions de compétence du tribunal, fortes ont été les critiques mues par une utilisation de l'arbitrage contraire à ce postulat de départ. L'exemple le plus évident en est la question de l'emploi de la clause de la nation la plus favorisée en matière procédurale $^{121}$ : l'investisseur n'ayant pas un droit fondamental à la saisine du tribunal arbitral, les dispositions procédurales sont considérées par une partie des arbitres comme étant à distinguer foncièrement des clauses de protection matérielle et devant être comprises à l'aune du principe de justice consensuelle. ${ }^{122} \mathrm{La}$ tendance jurisprudentielle opposée part du présupposé, inconciliable avec l'essence consensuelle de l'arbitrage d'investissement, selon lequel l'accès au tribunal arbitral fait partie intégrante de la protection internationale de l'investisseur et en est même la pierre angulaire.

En poussant à l'extrême le discours du droit au tribunal arbitral, on parviendrait à accepter l'hypothèse, explorée par certains, que l'offre d'arbitrage elle-même devienne coutumière, ce qui transformerait l'arbitrage d'investissement en un contentieux obligatoire généralisé que l'État n’a pas à accepter à l'avance, mais qui s'impose à lui. En présence d'une telle règle, "State consent would be deemed to be established on the basis of an unwritten rule ${ }^{123}$ Les professeurs Audit et Forteau, ayant étudié cette hypothèse, reconnaissent que tel n'est pas l'état du droit actuel, mais y voient une possible évolution progressive du contentieux préliminaire et considèrent qu'une telle coutume est in statu nascendi. ${ }^{124}$ Cette hypothèse, aussi spéculative qu'elle puisse être, appelle deux ordres de perplexité. Premièrement, force est de constater qu'une telle règle renverserait complètement la logique consensuelle qui gouverne le contentieux international aujourd'hui : on serait face à un droit coutumier de l'investisseur au tribunal arbitral, un « right to international arbitration that any investor could acti-

121 À ce sujet E. Stoppioni, The Jurisdictional Impact of MFN Clauses, MPILux Working Paper 3, 2017, 26 p.

122 Voir sur ce point l'opinion dissidente de B. Stern précitée et celle de L. Boisson de Chazournes dans Garanti Koza LLP c. Turkmenistan, affaire CIRDI $n^{\circ}$ ARB/11/20 du 3 juillet 2013.

123 M. Audit et M. Forteau, Investment Arbitration without BIT : Toward a Foreign Investment Customary Based Arbitration?, 29(5) Journal of International Arbitration (2012), 585.

124 Ibid., p. 586-590. 
vate against any State ${ }^{125}$ qui viendrait se substituer à un "principle of State consent in inter-State disputes [that] is not entirely relevant within the framework of the law of foreign investments $» .{ }^{126}$ Nous avons démontré ailleurs que l'optique du droit au juge sied mal au contentieux de droit international public ${ }^{127}$ et on voit mal en quoi elle siérait plus à l'arbitrage transnational. Deuxièmement, il nous semble délicat d'affirmer qu'il existe une pratique générale acceptée comme étant de droit en ce sens, surtout à une époque où la pratique conventionnelle des États va dans le sens inverse. Il suffit de penser aux États d'Amérique latine dont certains n'ont jamais voulu insérer de clause de règlement des différends dans leurs TBI (comme le Brésil) et d'autres sont en train de dénoncer la Convention de Washington pour ne plus être attraits devant des tribunaux arbitraux. On peut remarquer aussi la volonté des États de détailler toujours davantage, dans les nouveaux traités, les conditions posées dans la clause de règlement des différends à l'encontre d'une acceptation large du règlement arbitral des différends relatifs à l'investissement (il suffit de penser au nouveau modèle indien ${ }^{128}$ ou bien aux différents dispositifs contre le forum shopping dans le projet TAFTA $^{129}$ ).

En outre, et contrairement à ce que fait la Cour européenne, on ne saurait dégager des dispositifs d'arbitrages des obligations négatives ou positives de l'État d'accueil quant à l'accès de l'investisseur à l'arbitrage. Pour s'en convaincre, il suffit de se référer au cas particulier du consentement à l'arbitrage donné par l'État défendeur dans une loi nationale. Ici, l'État peut retirer à tout moment l'offre d'arbitrage formulée, ce qui devrait donc mettre un terme à la faculté procédurale de l'investisseur de saisir le tribunal arbitral. ${ }^{130}$ Cela ne veut pas dire que le comportement de l'État qui ferait obstacle au bon déroulement de l'arbitrage ne doive pas être sanctionné d'une manière ou d'une autre, mais la logique de l'obligation positive d'assurer la pleine jouissance du droit de l'investisseur à l'arbitrage ne semble pas une clef de lecture pertinente.

125 Ibid., p. 587.

126 Ibid., p. 597.

127 Stoppioni, supra note 121.

128 Voir le très détaillé article 14 concernant le règlement des différends dans le TBI modèle de 2015.

129 Voir le projet de la Commission européenne concernant d'articles 14 et 15 TAFTA.

130 Voir la très intéressante opinion dissidente de B. Stern dans l'affaire ABCI Investments N.V. c. République de Tunisie, affaire CIRDI no ARB/04/12, opinion du 14 février 2011. 


\section{Deux analyses différentes de l'origine de la protection internationale}

Les différences dans la conception globale de la nature de l'accès se reflètent également dans l'appréhension prétorienne de l'origine de la protection internationale : si le système de l'arbitrage d'investissement vise à protéger une personne privée située, le contentieux des droits de l'homme protège une personne soumise à la juridiction d'un État partie à la Convention. Dès lors que le seuil qui permet le déclenchement des obligations internationales est différent, la conception de l'accès à la juridiction internationale assume des contours différents.

Cette idée s'appuie sur la notion d'" investissement protégé ", dégagée dans la sentence Phoenix où le tribunal complète le test Salini avec les conditions de légalité et de bonne foi. ${ }^{131} \mathrm{La}$ décision adopte une approche téléologique de la protection internationale, ${ }^{132}$ en analysant la finalité de l'arbitrage d'investissement, pouvant opérer exclusivement dans l'optique d'accorder une protection internationale à un certain type de personne privée. L'investisseur n'est point protégé iure suo mais en raison de l'opération dont il est porteur et qui a une signification pour l'État d'accueil. Il doit être un opérateur économique, qui a investi de bonne foi dans l'économie locale en respectant les conditions d'accès au territoire de l'État d'accueil, que celui-ci a souverainement posées. De plus, la nationalité de l'investisseur est un élément central pour pouvoir jouir de la protection internationale, ${ }^{133}$ comme démontré notamment par le contentieux de l'abus de procédure consistant dans le changement de nationalité après l'émergence du litige dans le seul but de se ménager l'accès à l'arbitrage international. ${ }^{134}$

131 Phoenix Action Ltd. c. République Tchèque, affaire CIRDI n ARB/06/5, award, 15 April 2009, $\mathbb{S} 114$ : “To summarize all the requirements for an investment to benefit from the international protection of ICSID, the Tribunal considers that the following six elements have to be taken into account : 1 - a contribution in money or other assets; 2 - a certain duration; 3 - an element of risk; 4 - an operation made in order to develop an economic activity in the host State; 5 - assets invested in accordance with the laws of the host State; 6 - assets invested bona fide".

132 B. Stern, The Contours of the Notion of Protected Investment, 24(2) ICSID Review (2009), $534-551$.

133 U. Kriebaum, The Nature of Investment Disciplines, in Z. Douglas et al. (dir.), The Foundations of International Investment Law (2014) 47, 60, à comparer avec U. Kriebaum, Nationality and the Protection of Property under the European Convention on Human Rights, in I. Buffard et al. (dir.), International Law between Universalism and Fragmentation (2008), 649-666.

134 H. Ascensio, Abuse of Process in International Investment Arbitration, 13 Chinese Journal of International Law (2014), 763-770; pour une analyse récente et 
Tout au contraire, au sein du contentieux des droits de l'homme, la nationalité du requérant ne constitue pas une condition d'accès à la protection internationale. Celle-ci est remplacée par la notion de juridiction, clairement affirmé à l'article $1^{\text {er }}$ de la CEDH, puisque "Les Hautes Parties contractantes reconnaissent à toute personne relevant de leur juridiction les droits et libertés définis au titre I ", notion qui n'est pas circonscrite au territoire de l'État comme le démontre toute la jurisprudence sur l'extraterritorialité de la Convention. D’ailleurs, si l'on s'intéresse à la signification théorique de cette dernière, on peut retrouver les arguments mis en avant dans notre première partie. En effet, comme l'a démontré Samantha Besson, ${ }^{135}$ la compréhension que la Cour a, notamment depuis l'affaire $\mathrm{Al}$ Skeini, ${ }^{136}$ de la juridiction de l'État est celle de l'exercice d'une autorité politique et juridique, entraînant ainsi le devoir juridique de respecter les obligations en matière de droits de l'homme. Dans cette optique, " jurisdiction qua normative relationship between subjects and authorities actually captures the core of what human rights are about qua normative relationship between right-holders and institutions as duty bearers ». ${ }^{137}$

On peut conclure de cette analyse comparative de l'origine de la protection que le rapport qu'entretiennent l'État et la personne privée est différent dans les deux cas, dès lors qu'il puise sa source dans deux liens juridiques substantiellement et normativement distincts. Dans le contentieux des droits de l'homme, le recours individuel est motivé par la simple soumission d'une personne à l'autorité que l'État exerce dans sa juridiction et dont elle a été victime. Dans l'arbitrage d'investissement, le recours ne s'adresse qu'à un type particulier de personnes privées, en raison de leur instrumentalité pour une opération économique à laquelle l'État d'accueil a accepté au préalable d'accorder une protection internationale. C'est donc un autre argument illustrant que l'accès direct de l'investisseur à la juridiction internationale ne saurait être assimilé à un parangon unique. Sa nuance change avec toute une série de facteurs, parmi lesquels la physionomie du contentieux.

reprenant l'évolution prétorienne sur le sujet, voir Transglobal Green Energy, LLC and Transglobal Green Energy de Panama, S.A. c. République de Panama, affaire CIRDI $n^{\circ}$ ARB/13/28, sentence du 2 juin 2016, $\mathbb{S} 102-103$.

135 S. Besson, The Extraterritoriality of the European Convention on Human Rights : Why Human Rights Depend on Jurisdiction and What Jurisdiction Amounts to, 25(4) Leiden Journal of International Law (2012), 857-884.

136 Al-Skeini e.a. c. Royaume-Uni, CEDH arrêt du 7 juillet 2011, Requête $\mathrm{n}$ ${ }^{\circ} 55721 / 07$, notamment $\$ 130$.

137 Besson, supra note 135,860 . 
Le but principal de ce voyage à travers les discours jurisprudentiels autour de l'accès de la personne privée à la juridiction internationale a montré les potentialités d'une réflexion théorique et comparative pour contribuer à l'étude du droit international procédural, vocation première du département de l'Institut Max Planck dont la naissance est célébrée par cet ouvrage. Face à une branche du droit qui n'a pas encore atteint sa maturité, les analyses des grands théoriciens du procès de même que la comparaison des contentieux peuvent donc aider à structurer une grille d'analyse.

En effet, l'étude de l'étape initiale du contentieux international au sein des deux mécanismes semble révéler deux conceptions philosophiques différentes de l'accès direct à la juridiction internationale. Cette conclusion est possible en observant la morphologie des deux contentieux, dès lors que « ce n'est pas la volonté de l'auteur, du saisissant, qui produit l'obligation pour le juge d'examiner la demande, mais le système juridique luimême qui confere une qualité et un intérêt à agir à une personne physique ou morale pour accomplir cet acte et lui attache des effets de droit $\gg .138$

Reprendre les catégories des grands théoriciens du procès nous permet de concevoir la mesure dans laquelle la logique contentieuse des deux systèmes diverge. La Cour européenne voit dans l'article 34 de la Convention un droit subjectif de nature procédurale qui relève de la logique de protection des droits de l'homme propre à la Convention : clef de voûte de son fonctionnement, aucune réserve n'est admise à cette disposition, dont le juge fait découler des obligations positives à la charge de l'État. L’arbitre d'investissement ne se place pas dans cette optique d'humanisation de l'ordre juridique international et reste ancré à une logique davantage liée au consentement de l'État qui a admis souverainement l'opération économique sur son territoire.

Si le droit de recours individuel est conçu comme un droit fondamental par la Cour européenne des droits de l'homme, un tel discours s'adapte mal à l'architecture de l'arbitrage d'investissement. Dans ce cadre, on a pu isoler deux discours différents : celui de l'absence de droit inhérent et celui emprunté à l'idée d'un droit fondamental au tribunal arbitral. L'analyse qui précède a mis en avant que cette deuxième branche du discours arbitral procède d'une lecture contestable du système lui-même. Le mécanisme d'accès à l'arbitrage reste, en effet, une faculté procédurale instrumentale et déconnectée de la protection substantielle. 
